Special Issue of the 8th International Advances in Applied Physics and Materials Science Congress (APMAS 2018)

\title{
Complex Constitutive Characterizations of Materials in the X-Band Using a Non-Destructive Technique
}

\author{
T.A. Elwi ${ }^{a, *}$, A.J. SAlim ${ }^{b}$, A N. Alkhafaji ${ }^{b}$, J.K. Ali ${ }^{b}$ And A.S.A. JAlal ${ }^{c}$ \\ ${ }^{a}$ Department of Communication Engineering, Al-Mammon University College, Baghdad, Iraq \\ ${ }^{b}$ Microwave Research Group, Department of Electrical Engineering, University of Technology, Baghdad, Iraq \\ ${ }^{c}$ College of Information Engineering, Al-Nahrain University, 10070, Al-Jadriya Complex, Baghdad, Iraq \\ In this article, a measurement technique based on the Nicholson-Ross-Weir formulation to retrieve the complex \\ permittivity, $\varepsilon_{r}=\varepsilon_{r}^{\prime}+\mathrm{i} \varepsilon_{r}$, of materials from the measured $S$-parameters, $S_{11}$ and $S_{12}$, at the X-band, $8 \mathrm{GHz}$ up \\ to $12 \mathrm{GHz}$, is discussed. Finite element method simulation based on Comsol software package formulations is \\ invoked to evaluate the $S$-parameters based on the retrieved complex permittivity and to compare them to their \\ measurements. Then, a parametric study is conducted for the simulation to match the numerical results to their \\ identical measurements by considering the retrieved permittivity as an initial guess. Nevertheless, the complex \\ permittivity is measured using network/impedance material analyzer in the frequency range from $1 \mathrm{MHz}$ to $1.2 \mathrm{GHz}$ \\ to be compared against the evaluated values at the X-band. A PTFE sample is considered as an example to \\ validate the precision of the proposed method. The obtained $\varepsilon_{r}$ is found to be about 2.04-i0.0001, which is very \\ close to the manufacturer range. Finally, excellent agreement between the measured and simulated $S$-parameters \\ is observed
}

DOI: 10.12693/APhysPolA.135.567

PACS/topics: FEM, NRW, PTFE

\section{Introduction}

Identifying the material properties in the microwave range has received a great interest in the aerospace and telecommunication industries [1]. During last few years, several techniques were investigated to measure the relative permittivity of materials [2-5]. The most common methods are cavity perturbation, free-space measurement, and closed waveguide techniques. The freespace technique is employed for characterizing material samples, under the test of large dimensions [2]. However, this technique involves undesirable reflections from the surrounding circumstances, difficulties of impinging a plane wave in a limited area, and diffractions from the edges of the sample that lead to low measurement precision [3]. Therefore, using focusing lenses in such techniques are applied to reduce the reflections and diffraction effects [4]. In the cavity perturbation techniques or resonant cavity measurements, the precision is more accurate than the free space technique [5], but these measurements are only applicable for a limited frequency band, where this technique acquires very sensitive design parameters for the frequency band of interest [6]. Closed waveguide method is widely used in complex permittivity measurements of materials with wide frequency bands [7]. Despite the fact, this method has lower accuracy than the resonant cavity method [8], in which the analysis at the dominant mode is appropriate with high accuracy of retrieving the complex permittivity. However, the accuracy

*corresponding author; e-mail: taelwi82@gmail.com of extracting the complex permittivity is very sensitive to the uniformity of the cross-sectional area of the sample under test, as well as air gap defects [9].

In this study, the PTFE is used as a reference sample to validate the applied the Nicholson-Ross-Weir (NRW) technique and the finite element method (FEM) simulations for retrieving the complex permittivity of materials. The retrieved complex permittivity is obtained after running the measured S11 and S12 spectra from a rectangular waveguide in the NRW formulations at the $\mathrm{X}$-band. This technique is applied to a low loss material, which can be extended to other types of materials. Furthermore, FEM simulations are conducted to validate the retrieved complex permittivity of the sample from matching the measured $S$-parameters to the simulated results. The comparison of the simulation using FEM and measured $S$-parameters is provided for further confirmation of the obtained complex permittivity. The rest of this work is organized as follows: in Sect. 2, the material preparation and experimental methodology are discussed. The results are presented in Sect. 3. Finally, the paper is concluded in Sect. 4.

\section{Material and methods}

PTFE as a reference sample, of $1 \mathrm{~mm}$ in thickness, is loaded inside a WR-90 rectangular waveguide followed by measurement of the $S$-parameters at the X-band using Agilent 8720 network analyzer as seen in Fig. 1. It is worth noting that the sample must fully fit the crosssectional area of the waveguide with a minimum air gap to avoid any additional losses due to defects. The fundamental transverse electromagnetic (TE10) mode is the only applied mode in this measurement. The network 

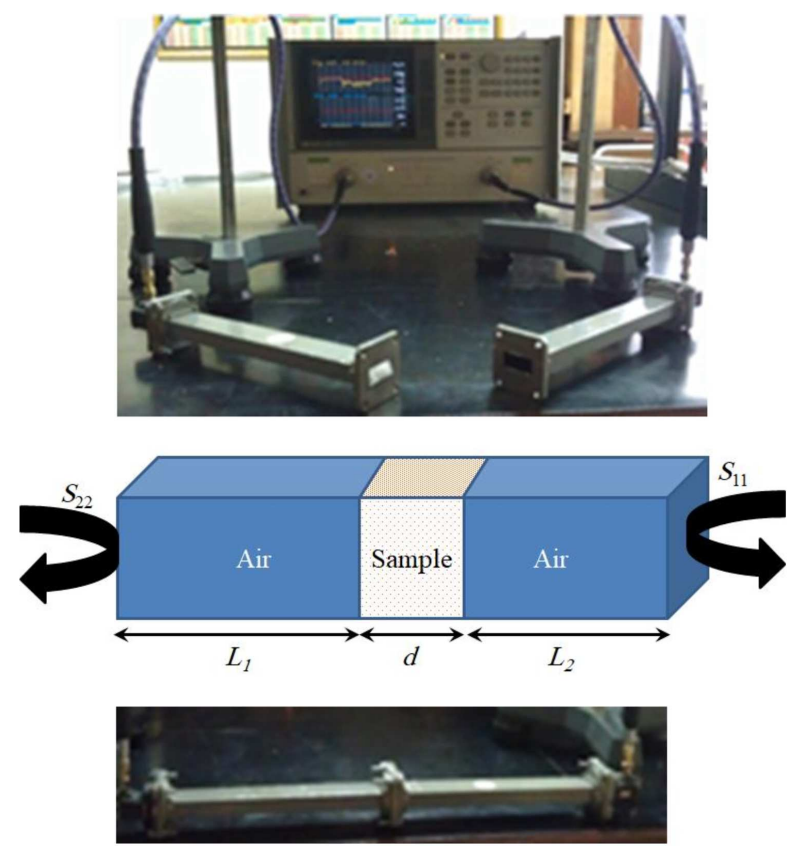

Fig. 1. $S$-parameters measurements setup using a WR-90 rectangular waveguide.

analyzer is calibrated using two-port calibration method (reflection, transmission, through, and omit isolation) for 201 frequency steps.

In NRW method, the reflection and transmission coefficients are calculated for the sample by applying the measured $S$-parameters from the network analyser to Eqs. (1) and (3). Then, as seen in the flowchart presented in Fig. 2, the complex permittivity can be retrieved using Eq. (4). In these calculations, the reflection coefficient must be less than unity. Therefore, the corresponding root that satisfies Eq. (1) can be obtained from Eq. (2). The real part of the retrieved permittivity, indeed, is measured from corresponding delay in the electromagnetic wave relative to air, while the imaginary part is attributed from the stored energy in the crystal lattice of the material. Therefore, the length of the sample is very effective parameter during the calculation of the complex permittivity using NRW method. Nevertheless, in our followed procedure, the authors used $1 \mathrm{~mm}$ sample thickness for the following reasons: firstly, most printed circuit boards for microwave and telecom industries are in the range of slim thicknesses; therefore, as a subject of factual fabrication $1 \mathrm{~mm}$ sample is selected. Secondly, with the aim of minimizing the relative error in the retrieving processes, in the worst case, a sample of $1 \mathrm{~mm}$ thickness is considered. Thus, the retrieved complex permittivity can be achieved with minimum error. The complex permittivity of other thicknesses, dramatically, can be evaluated in the suggested process of this paper

$$
\Gamma=X \pm\left(X^{2}-1\right)^{\frac{1}{2}}
$$

where $|\Gamma|<1$ is required for finding the correct root and in terms of $S$-parameters [10]:

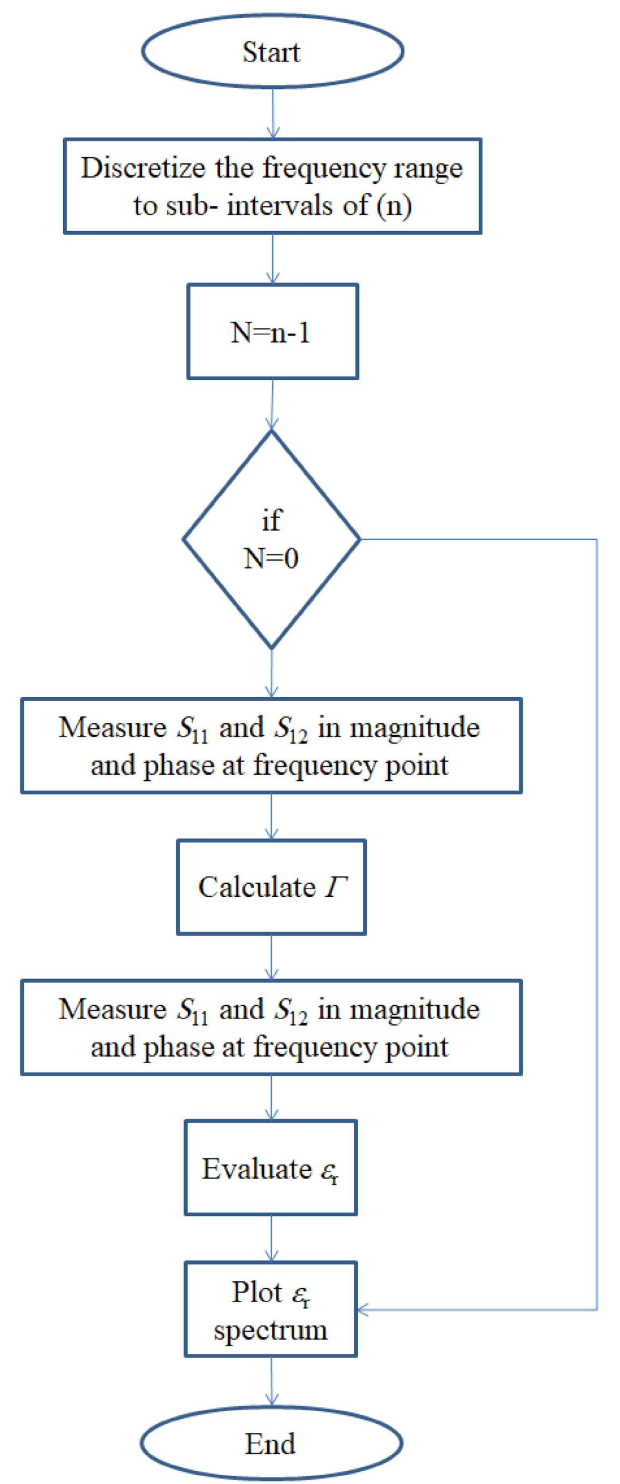

Fig. 2. The complex permittivity retrieving procedure.

$$
X=\frac{S_{11}^{2}-S_{21}^{2}+1}{2 S_{11}} .
$$

The transmission coefficient can be calculated from

$$
T=\frac{S_{11}+S_{21}-\Gamma}{1-\left(S_{11}+S_{21}\right) \Gamma} .
$$

Therefore, the permittivity can be calculated from

$$
\varepsilon_{r}=-\left[\frac{C}{w d} \ln \left(\frac{1}{T}\right)\right]^{2} .
$$

As an alternative scenario, subsequently, a nonattendant approximation processes based on finite element method, Comsol software package [11], is invoked in order to validate the retrieved complex permittivity from NRW technique. The FEM simulation is performed inside a rectangular waveguide with the same specifications and dimensions that were used in the measurements. The TE10 mode is excited by assigning wave ports. The input complex permittivity of the sample is assumed to 
be of the same value retrieved from the NRW. The evaluated results, in terms of $S$-parameters from the FEM simulation, are extracted to be from $8 \mathrm{GHz}$ to $12 \mathrm{GHz}$. An intensive convergence criteria, with minimum errors between two consecutive iterations not exceeding $1 \%$, is assigned.

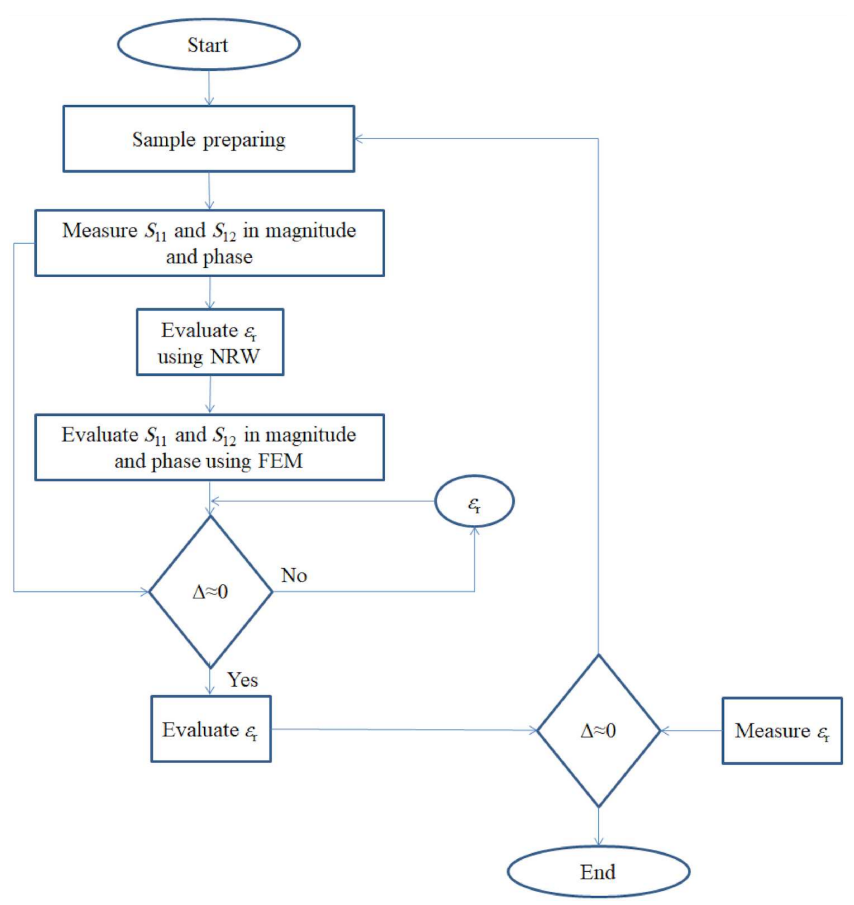

Fig. 3. Flowchart represents complex permittivity retrieving procedure using NRW and FEM.

In addition, Agilent 4291B RF impedance/material analyzer is introduced to measure the complex permittivity using capacitor technique in the frequency range from $1 \mathrm{MHz}$ to $1.8 \mathrm{GHz}$ as measured values for further comparisons with the retrieved complex permittivity using both NRW and FEM methods. Figure 3 shows a flowchart that represents the procedure of $S$-parameters measurements and complex permittivity retrieving.

\section{Results and discussion}

The measured $S$-parameters and retrieved complex permittivity for the PTFE sample using the WR-90 rectangular waveguide with Agilent 8720 network analyzer at the X-band are discussed. As can be noted from Fig. 4, insignificant ripples appear in the measured $S$ parameters which attributed to the multipath reflections inside the waveguide and the surface of the sample. However, the simulated results show an excellent agreement with the measured results after considering the same retrieved complex permittivity from the NRW in the FEM simulation. The calculated relative errors of the FEM corresponding to the measured $S$-parameters are found to be not exceeding 0.121 and 0.093 for $S_{11}$ and $S_{12}$, respectively.

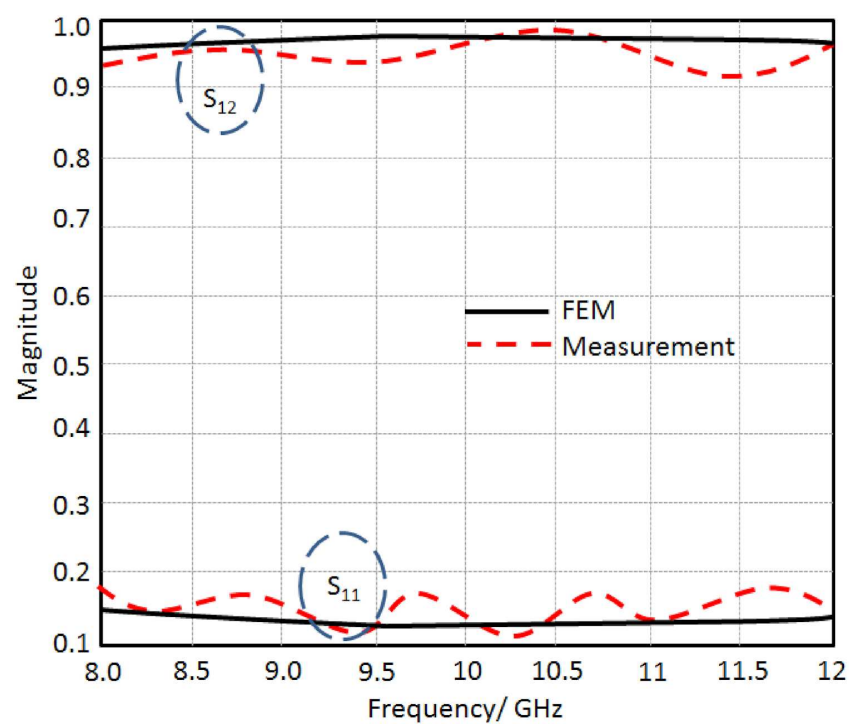

Fig. 4. The measured and simulated magnitude of $S_{11}$ and $S_{12}$ at X-band for PTFE with a thickness of $1 \mathrm{~mm}$.

TABLE I

Measured, retrieved (NRW, FEM) and manufactured complex permittivity of PTFE only.

\begin{tabular}{l|c|c}
\hline \hline \multicolumn{1}{c|}{ Technique } & Real part & Imaginary part \\
\hline manufacturing & 2.04 & 0.001 \\
NRW & 1.9 & 0.0018 \\
FEM & 2.01 & 0.0013 \\
measurements & 2.07 & 0.001
\end{tabular}

As listed in Table I, the sample shows a real part of the retrieved complex permittivity of 1.9, while the imaginary part is 0.0018. As mentioned above, the retrieved results from the NRW are applied in the FEM simulation as initial guess to extract the $S$-parameters numerically. Therefore, a parametric study based on FEM simulation is investigated to match the $S$-parameters from numerical simulations to the measured results. To arrive to the best matching between the simulated and measured $S$ parameters, the complex permittivity is found to be at $2.01 \pm i 0.0013$. Moreover, the measured permittivity at the low band of frequencies shows minor deviation from the evaluated and retrieved results at the X-band as presented in Table I. It can be noted that the retrieved values of complex permittivity using both techniques, the NRW and FEM, are well agreed with recommended data by the manufacturing of PTFE sample as listed in Table I.

\section{Conclusion}

A rectangular sample of $1 \mathrm{~mm}$ PTFE positioned inside a rectangular waveguide to characterize the complex permittivity using NRW and FEM simulation is investigated in this paper at the X-band. The measured $S$-parameters of the PTFE sample are used with NRW method to retrieve the complex permittivity. The retrieved complex permittivity is assigned to the material structure 
inside the FEM simulation to obtain the $S$-parameters numerically. After running a parametric study in the FEM simulation while changing the complex permittivity around the retrieved value, an acceptable comparison is achieved between measured and simulated $S$-parameters. The complex permittivity at low frequencies is measured and compared to the evaluated and retrieved values of the complex permittivity. The maximum relative error between the measured and simulated results is found to be 0.12 for different values of $S_{11}$ parameters. This is due to the fact of surface roughness of the sample as well as the difficulty of achieving normal incident mode inside the waveguide, either due to the alignment of the sample with the faces of the waveguide or slope of the sample face. Finally, it is found that the evaluated complex permittivity from the FEM shows the best matching with recommended values by the manufacturer.

\section{Acknowledgments}

The authors gratefully acknowledge the Department of Physics at the University Putra Malaysia for their support during the simulation and measurement process. Moreover, the acknowledgment is extended to the Institute of Advanced Technology, University Putra Malaysia for using their laboratory facilities during sample preparing.

\section{References}

[1] T.A. Elwi, Sci. Rep. 9, 2174 (2019).

[2] Y. Al-Adhami and E. Erçelebi, in: Proc. 2018 Third Scientific Conference of Electrical Engineering (SCEE), Baghdad, Iraq, 2018, p. 29.

[3] T.A. Elwi, Prog. Electron. Res. Lett. 34, 1 (2012).

[4] T.A. Elwi, Al-Ma'mon College J. 2016, 262 (2016).

[5] A.R. Azeez, T.A. Elwi, Z.A. Abed AL-Hussain, Eng. Sci. Technol. Int. J. 20, 1140 (2016).

[6] T.A. Elwi, Y. Alnaiemy, Diyala J. Pure Sci. 11, 1 (2015).

[7] T.A. Elwi, M.M. Hamed, Z. Abbas, M.A. Elwi, Int. J. Electron. Commun. 68, 846 (2014).

[8] T.A. Elwi, B.A. Ahmed, AEU - International Journal of Electronics and Communications 96, 122 (2018).

[9] T.A. Elwi, H.M. Al-Rizzo, D.G. Rucker, E. Dervishi, Z. Li, A.S. Biris, Nanotechnology 21, 1 (2010).

[10] H.S. Ahmed, T.A. Elwi, Int. J. Electron. Lett. 7, 236 (2019).

[11] Comsol Multiphysics Engineering Simulation Software. 